\title{
Arthropathy in patients with hemophilia in the antananarivo hemophilia treatment center
}

\begin{abstract}
Hemophilia is a hereditary bleeding disorder related to $\mathrm{X}$ chromosome due to a coagulation factor deficiency. Sixty six patients with hemophilia $(\mathrm{PwH}) \mathrm{A}$ and fifty four patients with hemophilia B are until now registered at the HTC in Madagascar. The objective of this study is to describe the epidemioclinical and therapeutic profile of arthritis in Malagasy hemophiliacs at the HTC. This is a monocentric, retrospective and descriptive study extending over a period of 6 months from September 1, 2017 to March 31, 2018 including 30 cases of arthropathy. The age group between 11 and 20 and patients with hemophilia A severe were the most affected. Hemarthrosis has been frequent in 3 to 5 times a year. Arthropathy reached the knees in $70 \%$ of cases and has been debilitating in almost all of cases. Care has been essentially based on clot factor substitution. Repetition of hemarthrosis episodes leads to arthropathy which can be avoided by early infusion of concentrate clot factor.
\end{abstract}

Keywords: arthropathy, hemarhtrosis, hemophilia, antihemophilic factor, Madagascar
Volume 7 Issue 3 - 2019

\author{
Dinafanomezana Harijaona \\ Raymondine,' Rahantamalala Marie Ida, ${ }^{2}$ \\ Randriamampianina Tahianasoa, ${ }^{3}$ Vololontiana \\ Hanta Marie Danielle, ${ }^{4}$ Rakoto Alson Aimée \\ Olivat ${ }^{5}$ \\ 'Former Internal Clinical Hematology. Joseph Ravoahangy \\ Andrianavalona Hospital Center, Madagascar \\ ${ }^{2}$ Former Internal Medicine, Hospital Center Anosiala \\ Ambohidratrimo, Madagascar \\ ${ }^{3}$ Former Internal Clinical Hematology, Joseph Ravoahangy \\ Andrianavalona Hospital Center, Madagascar \\ ${ }^{4}$ Professor in Internal Medicine, Joseph Raseta Befelatanana \\ Hospital Center, Madagascar \\ ${ }^{5}$ Professor in biological hematology, Joseph Ravoahangy \\ Andrianavalona Hospital Center, Madagascar
}

\begin{abstract}
Correspondence: Donna Pitts L, Department of SpeechLanguage-Hearing Sciences, Loyola University Maryland Baltimore, MD 21210, USA, Email Dptts I @loyola.edu
\end{abstract}

Received: October II, 2019 | Published: November |4, 2019

\section{Introduction}

Hemophilia is a hereditary bleeding disorder related to $\mathrm{X}$ chromosome caused by a deficiency of coagulation factor; factor VIII for hemophilia A and factor IX for hemophilia B, almost exclusively found in males. The various degrees of deficit define the disease as being minor with a coagulation factor level between 6 to $40 \%$, moderate between 1 to $5 \%$ and severe less than $1 \%$. The prevalence of hemophilia A is estimated at 1 out of 5000 births, and hemophilia B is 1 out of 30000 births. ${ }^{1,2}$

Eighty-five percent of patients with hemophilia suffer from hemophilia A while $15 \%$ of them suffer from hemophilia B. But in Madagascar, there is almost as much hemophilia A as hemophilia B. The natural history of hemophilic arthropathy has been well described. Intra-articular bleeding causes chronic synovial hypertrophy and cartilage lesions, with gradual joint destruction according to Hilgartner, ${ }^{3}$ Roosendaal ${ }^{4}$ and Rodriguez. ${ }^{5}$ The treatment of hemophilia is based on intravenous injection of the deficient coagulation factor. ${ }^{6}$ Prophylactic treatment by regular injection of clotting factors is essential to prevent bleeding by delaying the development of hemophilic arthropathy. ${ }^{7}$ The aim of this study was to describe the epidemiological, clinical situation of hemophilic arthropathy in the Antananarivo Hemophilia Treatment Center.

\section{Patients and methods}

This is a single center, retrospective and descriptive study led from September 2017 to March 2018 at the Hemophilia Treatment Center in Antananarivo. Joint manifestations of Malagasy patients with hemophilia were sought during their visit to the center.
The orthopaedic evaluation was based on clinical and radiological examination. Radiological examination of the joints was evaluated according to the Pettersson scale ( $0-13$ points/joint).

The statistical analysis was performed using the Epi info 7 software. The results were given in the form of figures and tables and expressed as a percentage and on average.

\section{Results}

Thirty cases of arthropathy were collected from 150 hemophiliacs giving a prevalence of $20 \%$. The mean age for patients was 18 . The most affected age group was respectively 11 and 20 years old in $36.67 \%$ of cases, 1 and10 years then 21 and 30 years in $26.67 \%$ of each group, 31 and 40 years in $6.67 \%$ of cases and 41 and 50 years in $3.35 \%$ of cases.

Hemophilia A was dominant in $63.33 \%$ of cases and was severe in $90 \%$ of cases. More than half of the hemophilia patients involved $(\mathrm{n}=19.64 \%)$ had hemarthrosis between 3 and 5 times per year. Haemarthrosis was localized most often $(n=12.40 \%)$ at the knee level and without any other location (Figure 1). The locations at the elbow (Figure 2) and the ankle (Figure $3 \& 4$ ) were less frequent.

\section{Clinical aspect of hemarthrosis and arthropathy: from haemarthrosis to arthropathy}

In haemarthrosis, synovial resorbs hematological elements but less and less effective as the episodes multiply. Note the apparence of synovitis with thickening of the synovial membrane. In the arthropathy, we noted the alteration of the bone cartilage secondary to 
the intraarticular hyperpressure and the synovial hyperplasia causing erosions to the synovial reflexion zones.

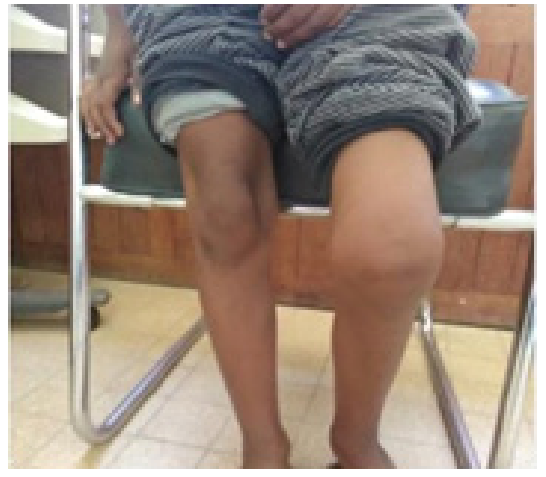

Figure I Hemarthrosis in the left knee.

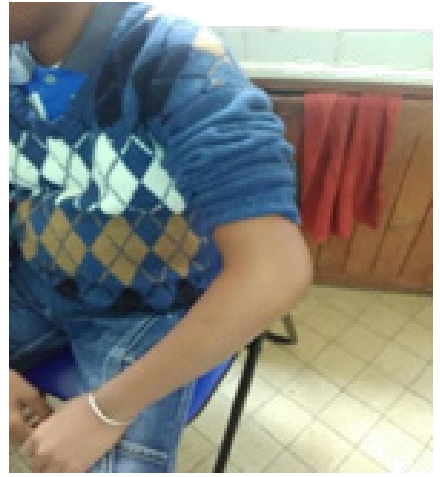

Figure 2 Hemarthrosis in the left elbow.

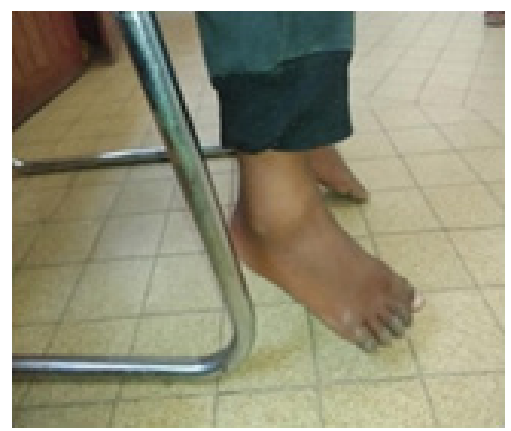

Figure 3 Hemarthrosis in the right ankle.

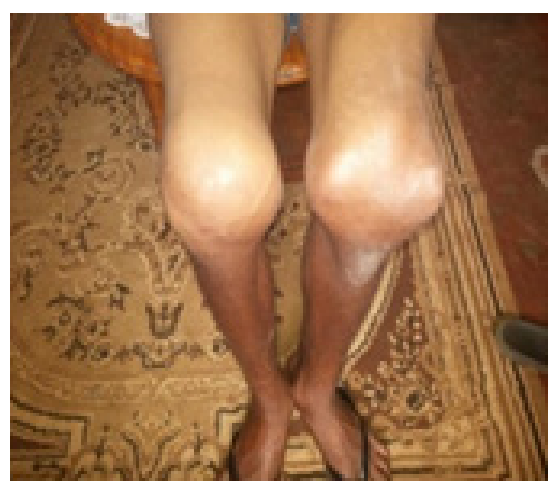

Figure 4 Arthropathy in the left knee.

\section{$\mathbf{X}$ - ray examination}

In the majority of cases, the imaging examination found soft tissue swelling with irregular bone contours (Figure $5 \& 6$ ), destruction of bone cartilage or even a fracture in $6.66 \%$ of cases. No cases of epiphyseal deformation, subluxation or ankylosis were found.
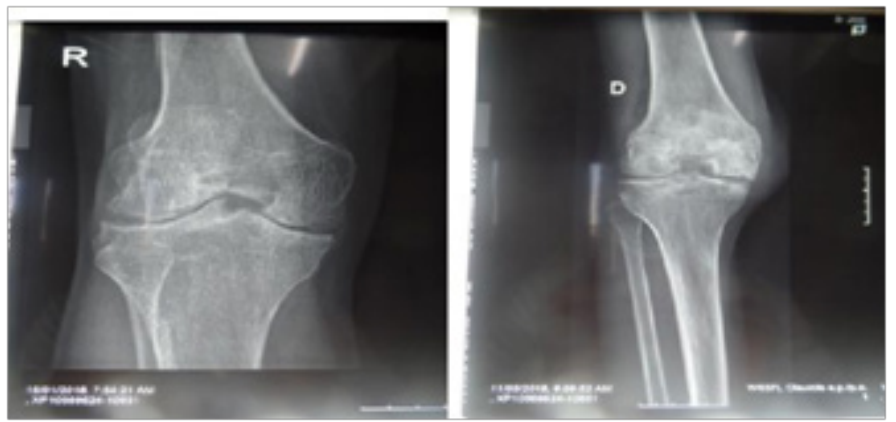

Figure 5 Irregularity of joint surfaces.

Figure 6 Joint incongruence.

Table 1 shows that all patients had benefited from replacement therapy with curative antihemophilic factor $(n=30,100 \%)$ and $83 \%$ $(\mathrm{n}=25)$ for prevention. $70 \%$ of patients $(\mathrm{n}=21)$ received fresh frozen plasma, $97 \%(\mathrm{n}=29)$ had functional rehabilitation and no surgery.

Table I Therapeutic means

\begin{tabular}{ll}
\hline Treatment & Percentage/ effective \\
\hline Antihemophilic coagulation factor & $100 \%(n=30)$ \\
Fresh frozen plasma & $70 \%(n=21)$ \\
Rehabilitation & $97 \%(n=29)$ \\
Surgery & $0 \%(n=0)$ \\
\hline
\end{tabular}

\section{Discussion}

Hemophilic arthropathy is painful, destructive and disabling. It can bring into play the functional prognosis in front of the handicap that it generates and especially the deterioration of the quality of life of the haemophiliacs. It predominates in the weight-bearing joints, especially the knee and ankle, less frequently in the hip. ${ }^{8}{ }^{9}$ Hemophilic arthropathy is the main complication of severe hemophilia. This is secondary to the repetition of hemarthrosis. A severe hemophiliac patient will present several episodes of haemarthrosis, spontaneously or after minor trauma. ${ }^{9}$ In Madagascar, no study was done on arthropathy in hemophiliacs, hence the interest of this study. This is a monocentric, retrospective, 5-month study that was performed at the CTH Hemophilia Treatment Center of the Joseph Ravoahangy Andrianavalona University Hospital from November 1, 2017 to March 31, 2018. Nevertheless, this study is limited by the small sample size that does not reflect the reality and the exact number of Malagasy hemophiliacs.

For the moment, hemophilia treatment centers and regional registries for tracking and identifying new cases of hemophilia need to be put in place. In addition, patients living in the provinces cannot regularly follow the recommendations from the center. That's why we did this study. We suggest the creation of provincial antennas for a better management of hemophiliacs as well as a perpetual follow-up of the treatment. 
Our study made it possible to collect 30 cases of hemophilic arthropathies over a period of 5 months. What constitutes a relatively important series of this hematological-pathological entity little studied. The high frequency found in our study is different from that reported by Windyga in Polande, ${ }^{10}$ showing 2269 hemophiliacs in 2 years with 23 cases of arthropathies giving a frequency of $1.01 \%$. This difference can be explained by the limit of the diagnostic means, especially that of the technical trays in our country, the inaccessibility to the care and the ignorance of this rare entity. We do not have regional centers to identify new cases and we mostly lack medical staff to be well trained in the practice of hemophilia care and regional offices, the correspondents are to be trained. Similarly, a Spanish study conducted by Lucia ${ }^{11}$ reported $45.4 \%(n=142)$ of arthropathies in severe hemophiliacs over a 1-year period.

The study conducted by Biss and colleagues ${ }^{12}$ in Canada in 2008 reported 2663 cases of hemophiliacs, $81 \%$ hemophiliac A $(n=2161)$ and $18 \%$ hemophilia $B(n=502)$. The average age of the patients in our sample was 18 years old with extremes of 5 and 47 years old. The age group 11 to 20 years predominated in $36.67 \%$ of cases; followed by that between 1 to 10 years and 21 to 30 years with a rate of $26.67 \%$ each. Boco $\mathrm{V}$ and colleagues ${ }^{13}$ reported 29 cases of hemophilic arthropathies in Cotonou with a mean age of 19 years. The study by Strauss and colleagues ${ }^{14}$ found a mean age of 30 years in 21 hemophiliacs with arthropathy over a 5-year period. Similarly, Windyga $^{10}$ found an average age of 26 years for hemophilia A and 23 years for hemophilia B with arthropathy. Other studies conducted by Molho and colleagues ${ }^{15}$ in 2000 found 116 cases of arthropathy in hemophiliacs with a mean age of 23 years, that reported by Aznar ${ }^{16}$ in Spain with a mean age of 22 years in 70 hemophiliacs presenting with arthropathy. Hemophilic arthropathy seemed to affect the young population in our study.

This could be explained by the fact that we lack therapeutic means to limit the occurrence of arthropathy. We do not manage to cover all of our patients in prevention and that is why arthropathy appears from a young age. During this study, we found that arthropathy mainly affects severe hemophilia; $90 \%$ of the sample. According to Barg and colleagues, ${ }^{17}$ arthropathy causes significant morbidity in severe hemophilic patients. Similarly, the study conducted by Windyga in Polande ${ }^{10}$ reported the prevalence of severe haemophilia in arthropathy; 59.7\% in hemophilia A and 56.6\% in hemophilia B. It could be said that arthropathy affects mainly severe hemophiliacs, so our study fits well with the literature data.

Thirty-seven percent of patients had more than three episodes of hemarthrosis per year, more than four episodes per year in $27 \%$ of cases, and more than five in $24 \%$ of cases. According to the literature, Lobet and colleagues ${ }^{18}$ as well as Stephensen ${ }^{19}$ found that hemarthrosis started mostly at walking age and that severe hemophilic patients had more than 30 hemarthroses per year to develop arthropathy. Nouffissa and colleagues ${ }^{20}$ reported 16 cases of haemophilia in 2014 with an average of 2- to 5-fold hemarthrosis in one year, and arthropathy in $33.3 \%$ of cases. The frequency of hemarthrosis was significantly influenced by the severity of hemophilia. This study is similar to the study conducted by Nouffissa ${ }^{20}$ but in this study arthropathy appears early compared to other studies described in the literature.

This could be explained by the fact that we often lack antihemophilic factor to practice prophylactic treatment from an early age to prevent the occurrence of arthropathy. We have anti-hemophilic factor donations from international agencies and that depends on the availability of factor supply, so we can not cover any episode of hemarthrosis in our patients.

Forty percent of our patients had knee osteoarthritis. Haemarthrosis has been described mainly in the knees according to Nouffissa ${ }^{20}$ and his collaborators. According to Lobet and colleagues ${ }^{18}$ hemarthrosis affects mostly the knees and elbows in subjects over 30 years of age, whereas they mainly affect the ankles in the very young. Diop and colleagues $^{21}$ reported that hemarthrosis is localized mainly in the knee, then the elbow and ankle. The susceptibility of these joints to hemarthrosis is explained by the fact that they have only one plane of mobility and that any stress outside this plane can lead to a capsulosynovial elongation source of haemorrhage. In this study, we reported the frequent location of hemarthrosis in the knee. Thus, our data are in good agreement with those of the literature.

$70 \%$ of patients had knee arthropathy. The study conducted by Windyga ${ }^{10}$ reported mainly the prevalence of arthropathy in the knee in 92 severe hemophiliacs. In contrast, Strauss and colleagues ${ }^{14}$ reported 21 cases of hemophiliacs with arthropathy in the elbow. This could be explained by the anatomical complexity of the elbow joint. Utukuri et al. ${ }^{22}$ also conducted 26 cases of elbow arthropathy in 2005 in London.

Similarly, Carulli and colleagues ${ }^{23}$ reported a case of arthropathy in a severe, age-related hemophiliac A, aged 46, and this one in the hip. Djaroud and colleagues ${ }^{24}$ also reported in 201111 cases of hemophiliacs including 4 cases of arthropathy in the shoulder. Meegener and colleagues ${ }^{25}$ reported 3 cases of arthropathy in the ankle in 2012. Our study is similar to Windyga's study in Polande ${ }^{10}$ and we find that arthropathy can reach all joints and joints basically at the knee. The frequency of onset of arthropathy in the knee could be explained by the fact that the knee is the body weight bearing joint. In this study, almost all patients had stage I arthropathy according to the Pettersson score, 1 stage II and one stage III with complete destruction of the joint.

According to Klukowska and colleagues, ${ }^{26}$ the radiographic appearance of hemophilic arthropathies is strongly correlated with the number of hemarthrosis episodes. Strauss and colleagues ${ }^{14}$ had 21 cases of hemophiliacs with an average Peterson score of 7.3 with irreversible but non-disabling injury that is stage II. Molho and colleagues ${ }^{15}$ also reported a Peterson score of 13.5 with massive joint destruction in 116 severe hemophilia patients. We still have limitations on coagulation factors because prophylaxis depends on the availability or not of the supply from the foreign body. Sometimes, for example, we encounter administrative problems concerning the customs clearance of products. But also, we are frequently faced with a break in medication. Thus, we suggest close collaboration with local or foreign organizations so that we can ensure the continuity of treatment (Figure 7).

During this study, $83.33 \%$ of patients had received prophylactic anti-hemophilic factor A or B treatment at a dose of $10 \mathrm{IU} / \mathrm{kg}$ body weight weekly. The study conducted by Taki ${ }^{27}$ in Japan reported that prophylactic treatment was indicated in hemophilia A, $51 \%$ of whom were severe, $11 \%$ moderate, but not indicated in the case of minor hemophiliacs. On the other hand, it has been indicated in haemophilia B, $44 \%$ of which are severe, $50 \%$ moderate and not indicated for minor haemophiliacs. In contrast, the study conducted by Lobet ${ }^{18}$ reported the indication of replacement therapy with antihemophilic factor in acute hemarthrosis. It is dosed at 25 to $30 \mathrm{IU} / \mathrm{kg}$ body weight with 2 
to 3 injections every 12 hours for hemophilia A and every 18 hours for hemophilia B. However, prophylactic treatment combines educational measures: wearing a haemophiliac card, good dental hygiene, proscription of anticoagulant treatments, gestures and activities at risk of haemorrhage and transfusion of concentrated pellets as a deficit factor. This binding treatment avoids the development of hemophilic arthropathies, but it is not yet widespread in Madagascar because of the high cost.

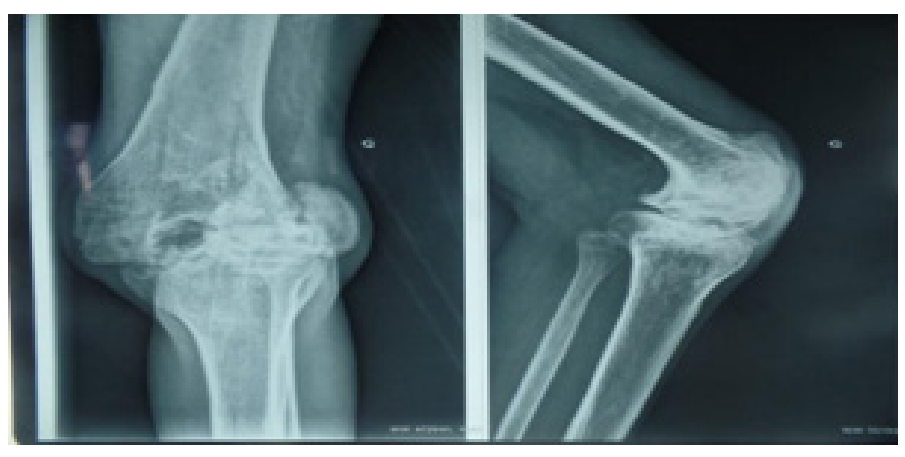

Figure 7 Erosion and joint destruction pronounced bone structure, fracture.

According to Rodriguez ${ }^{28}$ and Shupak, ${ }^{29}$ it has been shown that corticosteroid injection is effective in the treatment of hemophilic arthropathy. This relieves pain in $70 \%$ of cases. Shupak and colleagues $^{29}$ reported 10 cases of arthropathy injected with $80 \mathrm{mg}$ methylprednisolone. Similarly, Fernandez Palazzi et al. ${ }^{30}$ reported 34 cases of arthropathy treated with dexamethasone 3 cycles at 3-week intervals. They showed that intra-articular corticosteroid injection is effective in the medium term in the treatment of hemophilic arthropathy. According to Rattray and colleagues, ${ }^{31}$ they also showed the efficacy of anti-inflammatory drugs (Celecoxib) in arthropathy. The other therapeutic alternative would be intra-articular injection of hyaluronic acid according to Carulli et al. ${ }^{32}$ The latter will be produced by synovial cells and would be effective in delaying the development of chronic arthropathy.

For Wallny, ${ }^{33}$ five weekly injections of $50 \mathrm{mg}$ of hyaluronic acid reduce pain, increasing the walking and climbing ability of more than half of the patients, which is maintained at 26 months. None of our patients had benefited from treatment with corticosteroids or antiinflammatories. This could be explained by the lack of knowledge of the indication of corticosteroid in the treatment of arthropathy and especially the pecuniary problem of patients.

According to Lobet and colleagues, ${ }^{18}$ the treatment of hemophilic arthropathy consists mainly of conservative treatments such as physiotherapy, rehabilitation and arthrodesis. In this study, all patients had only benefited from functional rehabilitation on a weekly basis, especially in young hemophiliacs with progressive mobilization of the affected joint. The center at JRA Hospital has equipment for physiotherapy but moving to the center is expensive for patients. Surgical treatment is indicated when replacement therapy fails due to deficient factors. ${ }^{14}$

According to Lobet and colleagues, ${ }^{18}$ synovectomy or synoviorthesis is justified in the presence of recurrent hemarthrosis, mainly involving a single joint that may risk the occurrence of synovitis or chronic arthropathy. Total knee arthroplasty is the treatment of choice in patients with severe knee arthropathy according to Atkins and colleagues. ${ }^{34}$ Similarly, total hip arthroplasty is mandatory in patients with hip arthropathy to limit the various complications according to Carulli and colleagues. ${ }^{32}$ Concerning the elbow joint according to Alcalay and colleagues, ${ }^{35}$ the limitation of the joint must be prevented by the rigorous treatment of haemarthrosis, with the insertion of a posterior splint and even until resection of the radial head.

At the ankle, the alteration of one or other of the ankle joints can lead to programming a surgical or arthroscopic arthrodesis and that total ankle arthroplasty is a less secure solution, practiced by a small number of surgeons according to Takedani. ${ }^{36}$

None of our patients had received any surgical treatment because of the unavailability of surgical means and especially the pecuniary problem of our patients. Surgical procedures are expensive and require intermittent hospitalization.

\section{Acknowledgments}

None.

\section{Conflicts of interest}

The author declares no conflicts of interest.

\section{Funding}

None.

\section{References}

1. Franchini M, Mannucci PM. Past, present and future of hemophilia: a narrative review. Orphanet J Rare Dis. 2012;7:24

2. Rodriguez EC, Jimenez-Yuste V, Aznar JA, et al. Joint protection in haemophilia. Haemophilia. 2017;17 Suppl(2):1-23.

3. Hilgartner M. Current treatment of haemophilic arthropathy. Curr Opin Pediatr. 2002;14(1):46-49.

4. Roosendaal G, Lafeber FP. Pathogenesis of Haemophilic synovotis and arthropathy. The haemophilic joints. New Perspectives. Oxford, UK: Blackwell Publishing Ltd. 2003:12-16.

5. Rodriguez- Merchan EC. Pathogenesis, early diagnosis and prophylaxis for chronic haemophilic synovitis. Clin Orthop Relat Res. 1997;343:611.

6. Blanchette VS. Prophylaxis in the haemophilia population. Haemophilia. 2010;16 Suppl(5):181-188

7. Manco Johnson MJ, Abshire TC, Shapiro AD. Prophylaxis versus episodic treatment to prevent joint disease in boys with severe hemophilia. N England J Med. 2007;357:603-605.

8. Eichler D, Ehlinger M, Ambrosio A, et al. Arthrodèse de cheville chez le patient hémophilique. Rev Chir orthop et traumatol. 2017;103:876-881.

9. Rahme M, Ehlinger M, Faradji A, et al. Total knee arthroplasty in severe haemophilic patients under continuous infusion of clotting factors. Knee Surg Sports Traumatol Arthrosc. 2012;20(9):1781-1786.

10. Windyga J, Lopaciuk S, Stefanska E, et al. Haemophilia in Poland. Haemophilia. 2006;12(1):52-57.

11. Lucia JF, Aznar JA, Abad FL, et al. Prophylaxis therapy in haemophilia A: current situation in Spain. Haemophilia. 2011;17(1):75-80

12. Biss TT, Chan AK, Blanchette V. The use of prophylaxis in 2663 children and adults with haemophilia: results of the 2006 Canadian national haemophilia prophylaxis survey. Haemophilia. 2008;14(5):923-930. 
13. Boco V, Latoundji S, Zohoun I, Yekpe P. Les arthropathies hémophiliques à Cotonou. Med Afr Noire. 1997;44(3)153-156.

14. Strauss AC, Goldmann G, Ezzidin S, et al. Treatment options for haemophilic arthropathy of the elbow after failed conservative therapy: a single centre experience. Hamostaseologie. 2014;34 Suppl 1:S17-S22.

15. Molho P, Rolland N, Lebrun T, et al. Epidemiological survey of the orthopedic status of severe haemophilia A and B patients in France. Haemophilia. 2000;6(1):23-32.

16. Aznar JA, Magallon M, Querol F, et al. The orthopedic status of severe haemophiliacs in Spain. Haemophilia. 2000;6(3):170-176.

17. Barg A, Elsner A, Hefti D, et al. Haemophilia arthropathy of the ankle treated by total ankle replacement: a case series. Haemophilia. 2010;16:647-655

18. Lobet S, Hermans C, Lambert C. Optimal management of hemophilic arthropathy and hematomas. J Blood Med. 2014;5:207-218.

19. Stephensen D, Tait RC, Brodie N, et al. Changing patterns of bleeding in patients with severe haemophilia A. Haemophilia. 2009;15(6):12101214.

20. Nouffissa B, Yousra E, Mohammed A, et al. Hémophilie: état des lieux dans un service de pédiatrie dans la région de l'oriental du Maroc. Pan Afr Med J. 2014;18:126.

21. Diop S, Toure AO, Thiam D, et al. Profil évolutif de l'hémophilie A au Sénégal: étude prospective réalisée chez 54 patients. Transfusion clinique et biologique. 2003;10:37-40.

22. Utukuri M, Goddard J. Haemophilic arthropathy of the elbow Haemophilia. 2005;11(6):565-570.

23. Carulli C, Rizza A, Innocenti M. Hip arthropathy in Haemophilia. J Clin Med. 2017;6(4):44.

24. Djaroud Z, Terki K, Bensaber O, et al. Impact fonctionnel de l'arthropathie hémophilique. Annals of Physical and Rehabilitation Medicine. 2011;54S:96-99.

25. Meegener M, Veghel V, Kleijn P, et al. Joint destruction results in clinical and structural improvement of haemophilic ankle arthropathy: a series of three cases. Haemophilia. 2012;18(5):810-817.
26. Klukowska A, Czyrny Z, Laguna P, Brzewski M, Serafinkrol M, Rokicka R. Correlation between clinical, radiological and ultrasonographical image of knee joints in children with haemophilia. Haemophilia. 2001;7(3):286-292.

27. Taki M, Shirahata A. Current situation of regular replacement therapy (prophylaxis) for haemophilia in Japan. Haemophilia. 2009;15(1):7882.

28. Rodriguez C. Intraarticular corticosteroid injections in hemophilic arthropathy: are they recommended? Hosp Pract (1995). 2018;46(1):1-

29. Shupak R, Teitel J, Garvey MB, et al. Intraarticular methylprednisolone therapy in hemophilic arthropathy. Am J hematol. 1988;27(1):26-29.

30. Fernandez-Palazzi F, Caviglia HA, Salazar JR, et al. Intraarticular dexamethasone in advanced chronic synovitis in hemophilia. Clin Orthop Relat Res. 1997;(343):25-29.

31. Rattray B, Nugent DJ, Young G. Celecoxib in the treatment of haemophilic synovitis, target joints, and pain in adults and children with haemophila. Haemophila. 2006;12(5):514-517.

32. Carulli C, Felici I, Martini C, et al. Total hip arthroplasty in haemophilic patient with modern cementless implants. $J$ Arthroplast. 2015;30(10):1757-1760.

33. Wallny T, Brackmann HH, Semper H, et al. Intra articular hyaluronic acid for haemophilic arthropathy of the knee. In: Rodriguez- Merchan EL, Goddard NJ, editors. Musculoskeletal aspects of haemophilia, Oxford: Blackwell Science. 2000. p.61-65.

34. Atkins RM, Henderson NJ, Duthie RB. Joint contractures in the hemophilias. Clin Orthop Relat Res. 1987;219:97-106.

35. Alcalay M, Deplas A. Prise en charge rhumatologique de l'hémophilie (première partie : manifestations articulaires). Rev Rhum. 2002;69(9):868-876.

36. Takedani H, Kawasaki N, Abe Y, et al. Total ankle arthroplasty more than 10 years post operative follow up. Haemophilia. 2000;50:383. 